\title{
Introducing the new BTS Guideline: Management of non-tuberculous mycobacterial pulmonary disease (NTM-PD)
}

\author{
Charles S Haworth, ${ }^{1}$ Charles S Haworth, ${ }^{1}$ R Andres Floto ${ }^{1,2}$
}

\begin{abstract}
The new BTS Guidelines for the management of non-tuberculous mycobacterial pulmonary disease combine the best available evidence with expert consensus to generate a set of pragmatic Guidelines, published as a supplement to this issue of Thorax, to assist in the management of these challenging infections.
\end{abstract}

\section{RATIONALE FOR A NEW GUIDELINE}

Since the publication of the British Thoracic Society (BTS) Guideline on the 'Management of opportunistic mycobacterial infections' in $2000,{ }^{1}$ our understanding of the biology, epidemiology, diagnosis and management of lung infections caused by non-tuberculous mycobacteria (NTM) has advanced considerably, but there remains a lack of clinical trial data to direct practice and guide physicians. We have therefore sought to combine the best available evidence through systematic literature reviews, with expert consensus to generate a set of pragmatic guidelines, published as a supplement to this issue of Thorax, ${ }^{2}$ to assist in the management of these challenging infections. These guidelines do not cover the management of extrapulmonary infections or lung infections in neonates, infants or HIV-positive individuals.

This document is principally written for hospital-based specialists involved in the management of NTM pulmonary disease (NTM-PD) including respiratory and infectious disease physicians, paediatricians, immunologists, microbiologists and radiologists. However, we recognise that many other groups are involved in the multidisciplinary care of patients with NTM including general practitioners, pharmacists, specialist nurses and thoracic

\footnotetext{
Cambridge Centre for Lung Infection, Papworth Hospital, Cambridge, UK

${ }^{2}$ Department of Medicine, University of Cambridge, Cambridge, UK

Correspondence to Dr Charles S Haworth, Cambridge Centre for Lung Infection, Papworth Hospital, Cambridge CB23 3RE, UK; charles.haworth@ nhs.net
}

surgeons (and of course patients themselves), and we have incorporated their input and advice throughout the development of these guidelines.

\section{METHODOLOGY}

These guidelines were produced adhering strictly to criteria set by the AGREE collaboration $^{3}$ and to protocols described in The British Thoracic Society Standards of Care Committee guideline production manual. ${ }^{4}$ Briefly, clinical questions were structured in PICO (Patient, Intervention, Control, Outcome) format to define the scope of the guideline and direct the systematic literature searches. For each topic area, the following databases were searched (initially in November 2012 and then updated in June 2015) for relevant articles published in English since 1980: Ovid MEDLINE (including MEDLINE In Process), Ovid EMBASE and the Cochrane Library (including the Cochrane Database of Systematic Reviews, the Database of Abstracts of Reviews of Effects). The initial and second searches identified 7186 and 1687 abstracts, respectively, which were evaluated independently by two committee members (CSH, RAF) for relevance. Full articles of potentially relevant publications were then reviewed by at least two other committee members assigned to each guidelines section to appraise each of these papers independently using the Scottish Intercollegiate Guidelines Network critical appraisal checklists. Disagreements were resolved by discussion with the section partner and initial evidence statements and recommendations were developed. The full Guideline Committee met eight times between May 2012 and June 2015, and section teams also discussed progress regularly by email and teleconference.

Recommendations were developed and graded from A to D to indicate the strength of the supporting evidence. Important practical points learnt through clinical experience of managing NTM-PD but lacking any research evidence base were highlighted as 'Good Practice Points' (GPPs).
These draft recommendations and GPPs were then voted on by the Guidelines Committee using an anonymous electronic survey administered by the BTS project coordinator, with $80 \%$ or more agreement being the threshold for approval. While unanimous agreement was reached in most areas of the guideline, recommendations relating to the utility of drug susceptibility test results and some of the antibiotic treatment regimens were not agreed unanimously, reflecting the lack of high-quality data to direct management in these areas.

The BTS Standards of Care Committee (SOCC) reviewed the draft guidelines in November 2016, which following revision was made available in February 2017 for public consultation online and circulated to all the relevant stakeholders. The BTS SOCC then re-reviewed the revised draft guideline in June 2017, granting final SOCC approval for publication in July 2017.

\section{CHALLENGES TO GUIDELINE IMPLEMENTATION}

We believe that there are a number of current challenges to implementing NTM guidelines within the UK related to (1) how we define NTM pulmonary disease, (2) the impact of treating growing numbers of NTM infections on healthcare resources and (3) the reluctance of physicians to adopt new recommendations. We discuss these issues briefly below.

\section{Definition of NTM-PD}

A variety of different definitions have been used to describe the inflammatory lung disease associated with NTM infection, and the lack of a standardised definition nationally and internationally has hindered comparisons of epidemiology and trial outcome data, and the implementation of clinical practice guidelines. Furthermore, the licensing of medicines specifically for NTM-PD will require global randomised controlled trials using definitions and endpoints approved by international regulatory authorities. Thus, in the absence of evidence supporting use of an alternative definition, the Guideline recommends adoption of the American Thoracic Society/Infectious Diseases Society of America definition of NTM-PD. ${ }^{5}$

\section{Impact on healthcare resources}

The isolation of NTM from respiratory tract cultures ${ }^{6}$ and the prevalence of NTM-PD are increasing, which will have resource implications. Data from 
Medicare beneficiaries in the USA show that the prevalence of NTM-PD increased from 20 to 47 cases per 100000 persons between 1997 and 2007, an increase of 8\% per year. ${ }^{7}$ While there are differences in the prevalence and pathogenicity of NTM species within and between countries, similar increases in prevalence have been observed in Europe. A recent study from Germany analysed public statutory health insurance data of 125 patients with newly diagnosed NTM-PD and 1250 age, gender and comorbidity matched controls. The mortality rate for patients with NTM-PD and the control group in the 39-month observational period was $22.4 \%$ and $6 \%$, respectively $(\mathrm{p}<0.001)$; the mean direct expenditure per NTM-PD patient was $€ 39559$ (nearly fourfold higher than for a matched control); and hospitalisations were three times higher and accounted for $63 \%$ of the costs. ${ }^{8}$ Due to the complex nature of NTM-PD and the requirement for multidisciplinary care, the increased resource required to provide optimal guideline-based care may be challenging.

\section{Guidelines adoption}

A further challenge may be convincing physicians to integrate the new guideline into their clinical practice. A recent questionnaire survey involving a review of 1012 cases of NTM-PD in Europe reported that only $9.2 \%$ of patients treated for Mycobacterium avium complex (MACPD) received $>6$ months of a rifamycinethambutol-macrolide regimen $(17.8 \%$ in the UK, $8.4 \%$ in Spain, $8.0 \%$ in France, $7.6 \%$ in Italy and $4.3 \%$ in Germany). ${ }^{9}$ In a similar study performed in the USA, only $13 \%$ of patients with MAC-PD received treatment in line with American Thoracic Society/Infectious Diseases Society of America (ATS/IDSA) Guidelines. ${ }^{10}$ The apparent limited impact of guidelines on clinical practice may reflect lack of awareness or concerns about the potential complications of using multidrug treatment regimens.

One approach to improve standards of care for people with NTM-PD would be to develop regional centres with multidisciplinary input from specialists in respiratory medicine, infectious diseases, microbiology, radiology, immunology, thoracic surgery, pharmacology and dietetics. Such specialist NTM centres might particularly benefit individuals with (1) multidrug-resistant NTM such as Mycobacterium abscessus requiring treatment with prolonged hospital/domiciliary-based intravenous antibiotic regimens, (2) cavitary disease requiring surgical evaluation, (3) persistent or relapsing NTM infection or (4) coinfection with other bacteria and/or fungi.

\section{FUTURE RESEARCH}

There are many unanswered questions regarding NTM-PD diagnosis and management, and an important outcome of the guideline development process was to highlight gaps in knowledge. The NTM Guidelines Group identified six key questions for future NTM research to address:

1. What are the risk factors for developing NTM pulmonary disease?

2. What are the mechanisms contributing to patient-to-patient transmission of NTM?

3. Can we develop more rapid identification of NTM species from respiratory samples?

4. Can we better understand the role of drug susceptibility testing in predicting treatment outcomes?

5. Can we better understand the pathogenicity of specific NTM species and subspecies to facilitate treatment decision-making?

6. Are there more effective and more tolerable NTM treatment regimens?

The European NTM Registry, provided through the EMBARC European Bronchiectasis Registry platform, is due to be launched later this year (www.ntmlung. com) and will collect prospective data on patients with pulmonary NTM with the aim of better understanding the epidemiology, treatment approaches and long-term outcomes of individuals with NTM-PD in Europe. The registry will also help identify suitable patients for clinical trials of new drugs for NTM.

\section{SUMMARY}

The new BTS NTM Guidelines provide pragmatic management advice through combining the best available evidence with clinical experience. It is hoped that these guidelines will contribute to improved clinical outcomes for people with NTM-PD and provide a framework for future research into this condition.

Competing interests RAF has received funding from Insmed. CSH has received funding from Aradigm, Chiesi, Gilead, Insmed, Raptor, Teva, Vertex, Zambon.

Provenance and peer review Commissioned; internally peer reviewed.

(C) Article author(s) (or their employer(s) unless otherwise stated in the text of the article) 2017. All rights reserved. No commercial use is permitted unless otherwise expressly granted.

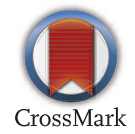

To cite Haworth CS, Haworth CS, Floto RA. Thorax 2017;72:969-970.

Thorax 2017;72:969-970.

doi:10.1136/thoraxjnl-2017-210929

\section{REFERENCES}

1 Subcommittee of the Joint Tuberculosis Committee of the British Thoracic Society. Management of opportunist mycobacterial infections: Joint Tuberculosis Committee Guidelines 1999. Thorax 2000;55:210-8.

2 British Thoracic Society Guidelines for the management of non-tuberculous mycobacterial pulmonary disease (NTM-PD). Thorax 2017;72(S2):ii1-ii55.

3 AGREE Advancing the science of practice guidelines. www.agreetrust.org/resource-centre/agree-ii/

4 British Thoracic Society. http://www.brit-thoracic.org. uk/guidelines-and-quality-standards/

5 Griffith DE, Aksamit T, Brown-Elliott BA, et al. An official ATS/IDSA statement: diagnosis, treatment, and prevention of nontuberculous mycobacterial diseases. Am J Respir Crit Care Med 2007;175:367-416.

6 Shah NM, Davidson JA, Anderson LF, et al. Pulmonary Mycobacterium avium-intracellulare is the main driver of the rise in non-tuberculous mycobacteria incidence in England, Wales and Northern Ireland, 2007-2012. BMC Infect Dis 2016;16:195.

7 Adjemian J, Olivier KN, Seitz AE, et al. Prevalence of nontuberculous mycobacterial lung disease in U.S. Medicare beneficiaries. Am J Respir Crit Care Med 2012;185:881-6.

8 Diel R, Jacob J, Lampenius N, et al. Burden of nontuberculous mycobacterial pulmonary disease in Germany. Eur Respir J 2017;49:1602109.

9 van Ingen J, Wagner D, Gallagher J, et al. Poor adherence to management guidelines in nontuberculous mycobacterial pulmonary diseases. Eur Respir J 2017;49:1601855.

10 Adjemian J, Prevots DR, Gallagher J, et al. Lack of adherence to evidence-based treatment guidelines for nontuberculous mycobacterial lung disease. Ann Am Thorac Soc 2014;11:9-16. 